\title{
Asteroseismology of red giants from the first four months of Kepler data: Fundamental stellar parameters
}

\author{
T. Kallinger ${ }^{1,2}$, B. Mosser ${ }^{3}$, S. Hekker ${ }^{4}$, D. Huber ${ }^{5}$, D. Stello ${ }^{5}$, S. Mathur ${ }^{6}$, S. Basu ${ }^{7}$, T. R. Bedding 5 , W. J. Chaplin ${ }^{4}$, \\ J. De Ridder ${ }^{8}$, Y. P. Elsworth ${ }^{4}$, S. Frandsen ${ }^{9}$, R. A. García ${ }^{10}$, M. Gruberbauer ${ }^{11}$, J. M. Matthews ${ }^{2}$, W. J. Borucki ${ }^{12}$, \\ H. Bruntt ${ }^{13}$, J. Christensen-Dalsgaard ${ }^{13}$, R. L. Gilliland ${ }^{14}$, H. Kjeldsen ${ }^{13}$, and D. G. Koch ${ }^{12}$
}

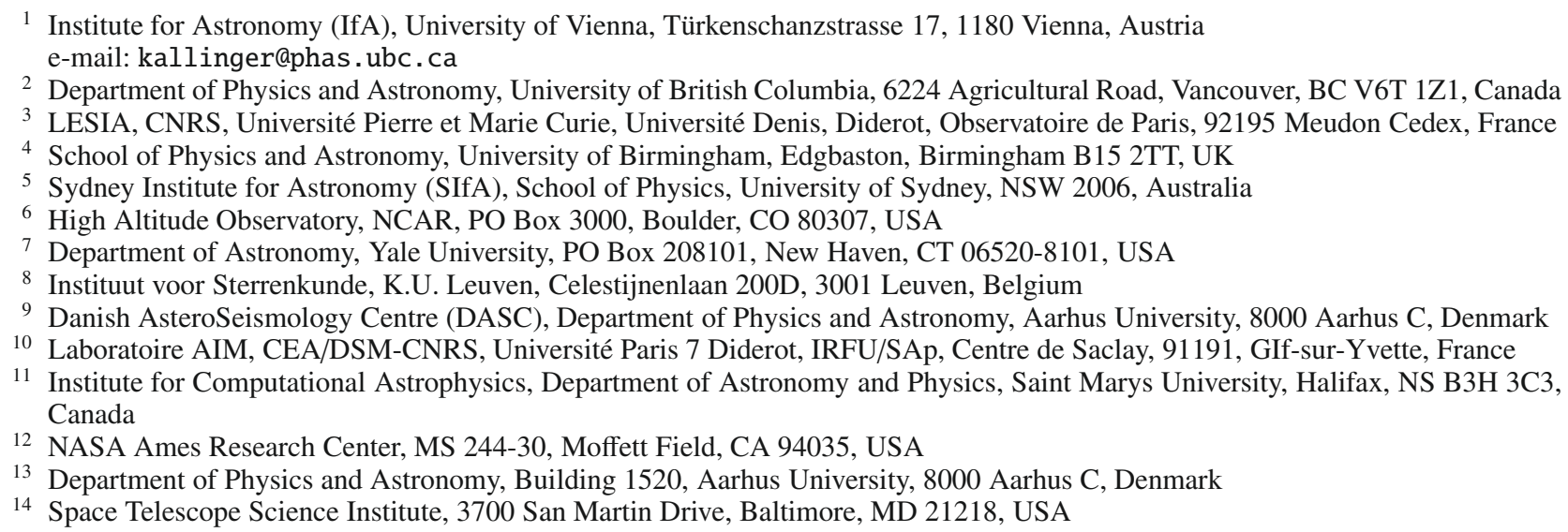

Received 23 June 2010 / Accepted 28 August 2010

\section{ABSTRACT}

Context. Clear power excess in a frequency range typical for solar-type oscillations in red giants has been detected in more than 1000 stars, which have been observed during the first 138 days of the science operation of the NASA Kepler satellite. This sample includes stars in a wide mass and radius range with spectral types $\mathrm{G}$ and $\mathrm{K}$, extending in luminosity from the bottom of the giant branch up to high-luminous red giants, including the red bump and clump. The high-precision asteroseismic observations with Kepler provide a perfect source for testing stellar structure and evolutionary models, as well as investigating the stellar population in our Galaxy.

Aims. We aim to extract accurate seismic parameters from the Kepler time series and use them to infer asteroseismic fundamental parameters from scaling relations and a comparison with red-giant models.

Methods. We fit a global model to the observed power density spectra, which allows us to accurately estimate the granulation background signal and the global oscillation parameters, such as the frequency of maximum oscillation power. We find regular patterns of radial and non-radial oscillation modes and use a new technique to automatically identify the mode degree and the characteristic frequency separations between consecutive modes of the same spherical degree. In most cases, we can also measure the small separation between $l=0,1$, and 2 modes. Subsequently, the seismic parameters are used to estimate stellar masses and radii and to place the stars in an H-R diagram by using an extensive grid of stellar models that covers a wide parameter range. Using Bayesian techniques throughout our entire analysis allows us to determine reliable uncertainties for all parameters.

Results. We provide accurate seismic parameters and their uncertainties for a large sample of red giants and determine their asteroseismic fundamental parameters. We investigate the influence of the stars' metallicities on their positions in the H-R diagram. Finally, we study the red-giant populations in the red clump and bump and compare them to a synthetic population. We find a mass and metallicity gradient in the red clump and clear evidence of a secondary-clump population.

Key words. stars: late-type - stars: oscillations - stars: fundamental parameters

\section{Introduction}

Studying solar-type oscillations has proved to be a powerful way to test the physical processes in stars (e.g. Christensen-Dalsgaard 2004) that are similar to our Sun and also to the more evolved red giants, which represent the future of our Sun. The turbulent motions in the convective envelopes of these stars produce an acoustic noise that can stochastically drive (and damp) resonant p-mode oscillations, typically with small amplitudes. On the other hand, the global properties of solar-type oscillations, such as the frequency range where they are excited to observable amplitudes and their characteristic spacings, are predominantly defined by the stellar mass and radius. By using accurate asteroseismic data, it should therefore be possible to constrain fundamental parameters to levels of precision that would otherwise be impossible. This has important applications in, for 
example, exoplanet studies, which depend on firm knowledge of the fundamental parameters of the host star. Asteroseismic data can put tight constraints on the absolute radii of transiting planets, or determine the age of an exoplanetary system (e.g. Christensen-Dalsgaard et al. 2010).

An obvious requirement for such asteroseismic studies is the availability of accurate observational data. The first indications of solar-type oscillations in $\mathrm{G}$ and K-type giants were based on ground-based observations in radial velocity (Arcturus: Merline 1999; $\xi$ Hya: Frandsen et al. 2002; $\epsilon$ Oph: De Ridder et al. 2006) and photometry (M 67: Stello et al. 2007), which largely suffered from low signal-to-noise data sets and aliasing. The periods of solar-type oscillations in red giants range from hours to days and hence call for long and preferably uninterrupted observations to resolve the oscillations, which can be done best from space. Space-based detection were made with the star tracker of the Wide Field Infrared Explorer satellite (WIRE; e.g. Buzasi et al. 2000; Retter et al. 2003), the Hubble Space Telescope (HST; e.g. Edmonds \& Gilliland 1996; Kallinger et al. 2005; Stello \& Gilliland 2009), Microvariability and Oscillation of Stars (MOST; Barban et al. 2007; Kallinger et al. 2008a,b), and the Solar Mass Ejection Imager (SMEI; Tarrant et al. 2007). Significant improvement in quality and quantity of the observations came from the 150-day long observations with the Convection, Rotation and planetary Transits satellite (CoRoT), which provided clear detections of radial and non-radial oscillation modes in numerous stars (De Ridder et al. 2009; Hekker et al. 2009; Carrier et al. 2010; Kallinger et al. 2010; Mosser et al. 2010). Most recently, the NASA Kepler Mission has demonstrated its great asteroseismic potential to observe solar-type oscillations in red giants (Bedding et al. 2010; Hekker et al. 2010b; Stello et al. 2010). We refer to our companion papers presenting a more detailed study of the asteroseismic observables (Huber et al. 2010) and a comparison of global oscillation parameters derived using different methods (Hekker et al. 2010c).

The oscillation spectrum of a solar-type oscillating star presents a pattern of modes with nearly regular frequency spacings, where the signature of these spacings carries information about the internal structure of the star. The large frequency spacing $(\Delta v)$, for example, is the frequency differences between consecutive overtones having the same spherical degree $(l)$, and is related to the acoustic radius and therefore to the mean density of the star (Brown et al. 1991; Kjeldsen \& Bedding 1995). Another directly accessible seismic parameter, the frequency of maximum oscillation power $\left(v_{\max }\right)$, is related to the acoustic cutoff frequency and therefore, in the adiabatic case and under the assumption of an ideal gas, defined by the surface gravity and effective temperature of the star (e.g. Kjeldsen \& Bedding 1995).

Estimating fundamental parameters from these seismic parameters has become an important application of asteroseismic observations. Recent investigations in this context were made by Stello et al. (2008), who analysed 11 bright red giants observed with the WIRE satellite. They compared traditional methods to determine stellar masses with a new method, that uses the effective temperature, the Hipparcos parallaxes and their measurement for $v_{\max }$, to estimate an asteroseismic mass. The A2Z pipeline (Mathur et al. 2010) uses two different methods to estimate the mass and radius of a star: one based on the scaling laws and the other one that starts with the measurement of $\Delta v$ and uses a pre-calculated grid of evolutionary models to obtain an initial guess of the fundamental parameters of the star. For the latter method, a minimisation algorithm is performed to estimate the radius and the mass with a higher accuracy
(Creevey et al. 2007). Basu et al. (2010) presented the YaleBirmingham (YB) method, which aims to deduce precise stellar radii from a combination of seismic and conventional variables. Within the context of the asteroFLAG hare-and-hounds exercises for the Kepler Mission, Stello et al. (2009b) summarised other methods, which provide stellar radii based on the observed large frequency separation and conventional observables. The basic principle of the YB and asteroFLAG methods is the same. They compare observed seismic parameters $\left(v_{\max }\right.$ and/or $\left.\Delta v\right)$ and other observables ( $T_{\text {eff }}, V, \pi, \log g$, metallicity, etc.) to those of stellar models, where the seismic parameters of the models are determined from scaling relations or adiabatic model frequencies. If the input parameters are well defined, these methods enable very precise estimates for the stellar radius. Most of the red giants observed with CoRoT and Kepler, however, are rather faint and, although the seismic parameters can be determined with high precision, additional constraints are generally very uncertain, if available at all. To account for this, Kallinger et al. (2010, hereafter Paper I) presented a modified approach for 31 red giants observed for about 150 days with the CoRoT space telescope. They exclusively used the measured seismic parameters $v_{\max }$ and $\Delta v$ to derive estimates for stellar fundamental parameters from the aforementioned scaling relations and a grid of solar-calibrated red-giant models, without making use of any other input parameters. They also indicated that their mass and radius determination is relatively insensitive to the metallicity and/or evolutionary stage of the investigated red giants.

In this paper, we largely follow the approach of Paper I but for red giants that have been observed during the first $\sim 138$ days of science operation of the Kepler satellite. We fit a global model to the power density spectra of the high-precision photometric time series to measure $v_{\max }$. We use an improved approach to determine the large and small frequency spacings, which also provides an automated identification of the mode degree. We apply the same methods to determine $v_{\max }$ and $\Delta v$ to SOHO/VIRGO (Variability of solar IRradiance and Gravity Oscillations; Frohlich et al. 1997) data to measure the solar reference values needed as an input for the scaling relations. We compare the measured seismic parameters with those determined from a multi-metallicity red-giant model grid (by using the scaling relations) to derive a reliable stellar mass and radius and a reasonable effective temperature and luminosity for the red giants in our sample. We investigate the error sources in our analysis and discuss the stellar populations on the giant branch.

\section{Observations}

The NASA Kepler Mission (Borucki et al. 2008, 2010) was launched in March 2009 with the primary goal of searching for transits of Earth-sized planets in and near the habitable zones of Sun-like stars. The satellite houses a $95-\mathrm{cm}$ aperture modified Schmidt telescope that points at a single field in the constellation Cygnus for the entire mission lifetime ( $>3.5$ years). The telescope feeds a differential photometer with a wide field of view that continuously monitors the brightnesses of about 150000 stars. This makes it an ideal instrument for asteroseismology and the Kepler Asteroseismic Science Consortium $(\text { KASC })^{1}$ has been set up to study many of the observed stars (see Gilliland et al. 2010, for an overview and first results).

In this paper we concentrate on the long-cadence (29.4 min sampling; Jenkins et al. 2010) data that have been collected within the astrometric and asteroseismic programmes during the

1 http://kepler.asteroseismology. com 
commissioning phase $(\mathrm{Q} 0 ; \sim 11 \mathrm{~d})$ and the first $(\mathrm{Q} 1 ; \sim 34 \mathrm{~d})$ and second $(\mathrm{Q} 2 ; \sim 89 \mathrm{~d})$ roll of the satellite. We analyse all stars for which at least Q1 and Q2 time series have been made available via KASC, including those which have not been flagged as red giants in the Kepler Input Catalogue (KIC; Latham et al. 2005). The combined time series consist of about 5900 or 5430 measurements and span a total duration of about 138 or 127 days, depending on the availability of Q0 data.

In Fig. 1 we show the relative flux for a typical red giant. Whereas the Q0 and Q1 time series show only long term trends, the Q2 data reveal a more complex behaviour. The time series appears to consist of five parts with sudden jumps at the transitions, which are instrumental. Additionally there is a step gradient at least at the beginning of the second and fourth "subset" (BJD $-2450000 \simeq 5018$ and 5062). These artefacts appear at the same time in most data sets and are due to some satellite failures during the Q2 observations, where Kepler had to be cooled down again after safe-mode operations. To account for these instrumental artefacts, we split the time series into 7 subsets (Q0, $\mathrm{Q} 1$, and 5 subsets for Q2). We tried several approaches to model the gradients, but this turned out to be quite difficult as the actual shape differed from star to star, even increasing gradients have been found in some cases. Therefore, we simply removed the leading data points, including the steepest part of the gradient for the first, second, and fourth subsets of the Q2 data. In total we rejected about 5.5 days of measurements, degrading the overall duty cycle from about 91 to $87 \%$, with only minor consequences on the spectral window function. Finally, we subtracted a second-order polynomial fit from each subset. The resulting time series is shown in the bottom panel of Fig. 1. This approach does, of course, suppress any intrinsic long period signal. The shortest subset is about 11 days long, which means that we filter out signal below about $1 \mu \mathrm{Hz}$.

The long-cadence data from Kepler that are accessible to KASC consist of two major samples. Firstly, the so-called astrometric reference stars (Batalha et al. 2010; Monet et al. 2010) comprising about 1000 stars that have been selected to be distant (and therefore having a small parallax), but bright (and therefore being mostly giants) and unsaturated stars in a Kepler magnitude range of 11.0-12.5 mag, which are uncrowded and uniformly distributed over the focal plane. Secondly, about 1300 stars that have been selected for asteroseismology by the various working groups of KASC according to different criteria such as their presumed membership to a cluster or due to their colour index. We computed the power density spectra between $1 \mu \mathrm{Hz}$ and the Nyquist frequency $(\sim 280 \mu \mathrm{Hz})$ for all stars and searched them visually (i.e., by eye) for red-giant characteristics. We found a total of 1041 stars (670 astrometric and 371 asteroseismic) that show both a clear power excess hump with regularly spaced peaks and a background that decreases towards higher frequencies. We identified them as red giants for the subsequent analysis.

\section{Power spectra modelling}

The power spectra of solar-type oscillations have characteristic features. Besides an instrumental white noise component, they show a frequency-dependent background signal. This signal can be represented by several super-Lorentzian functions ${ }^{2}$ with

\footnotetext{
2 Note that this function is frequently referred to as power law or Harvey-like model. It is, however, clearly not a power law and Harvey (1985) originally used a Lorentzian. We therefore suggest the name "super-Lorentzian" with the power 4, which is sometimes used in optics.
}

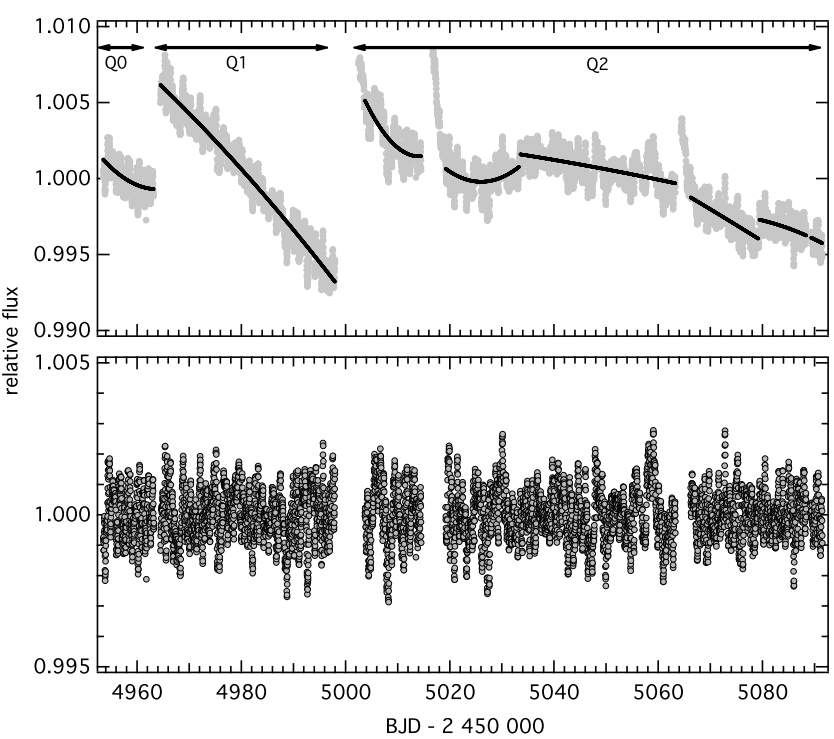

Fig. 1. Relative flux for the Q0, Q1, and Q2 data of KIC 6838420. The top panel shows the original time series (grey points) with the 2 nd order polynomial fits overlaid (black lines). The bottom panel shows the residual time series.

increasing characteristic frequencies and decreasing characteristic amplitudes. Each of these components is believed to represent a separate class of physical process such as stellar activity and the different scales of granulation, and most of them are strongly connected to the turbulent motions in the convective envelope. On top of the background signal, one finds additional power due to pulsation in a broad hump. This power excess arises from a sequence of stochastically excited and damped oscillations, which correspond to high-overtone radial and non-radial acoustic modes. The mode amplitudes are defined by the excitation and damping and are modulated by a broad envelope. The centre of the envelope is usually called the frequency of maximum oscillation power $\left(v_{\max }\right)$ and its shape is approximately Gaussian.

\subsection{Global power spectrum model and $v_{\max }$}

The background signal in the power spectra of solar-type oscillations can be modelled by the sum of super-Lorentzian functions, $P(v)=\sum A_{i} /\left(1+\left(2 \pi v \tau_{i}\right)^{c_{i}}\right)$, with $v$ being the frequency, $A_{i}, \tau_{i}$, and $c_{i}$ being the characteristic amplitudes, timescales, and the slopes of the background model. This model was first introduced by Harvey (1985) to characterise the solar background signal. In Paper I, it was shown that the solar background model also works for the power spectra of red giants. Due to the larger radii of red giants compared to the Sun, the amplitudes and timescales of the background components are quite different but the model, particularly the slopes of the components are the same. Here, we follow the approach of Paper I and model the observed power density spectra with a superposition of white noise, the sum of super-Lorentzian functions, and a power excess hump approximated by a Gaussian:

$P(v)=P_{\mathrm{n}}+\sum_{i} \frac{2 \pi a_{i}^{2} / b_{i}}{1+\left(v / b_{i}\right)^{4}}+P_{\mathrm{g}} \exp \left(\frac{-\left(v_{\max }-v\right)^{2}}{2 \sigma_{g}^{2}}\right)$

where $P_{\mathrm{n}}$ corresponds to the white noise contributions and $a_{i}$ is the rms amplitude of the $i$ th background components. The parameter $b_{i}$ corresponds to the frequency at which the power 


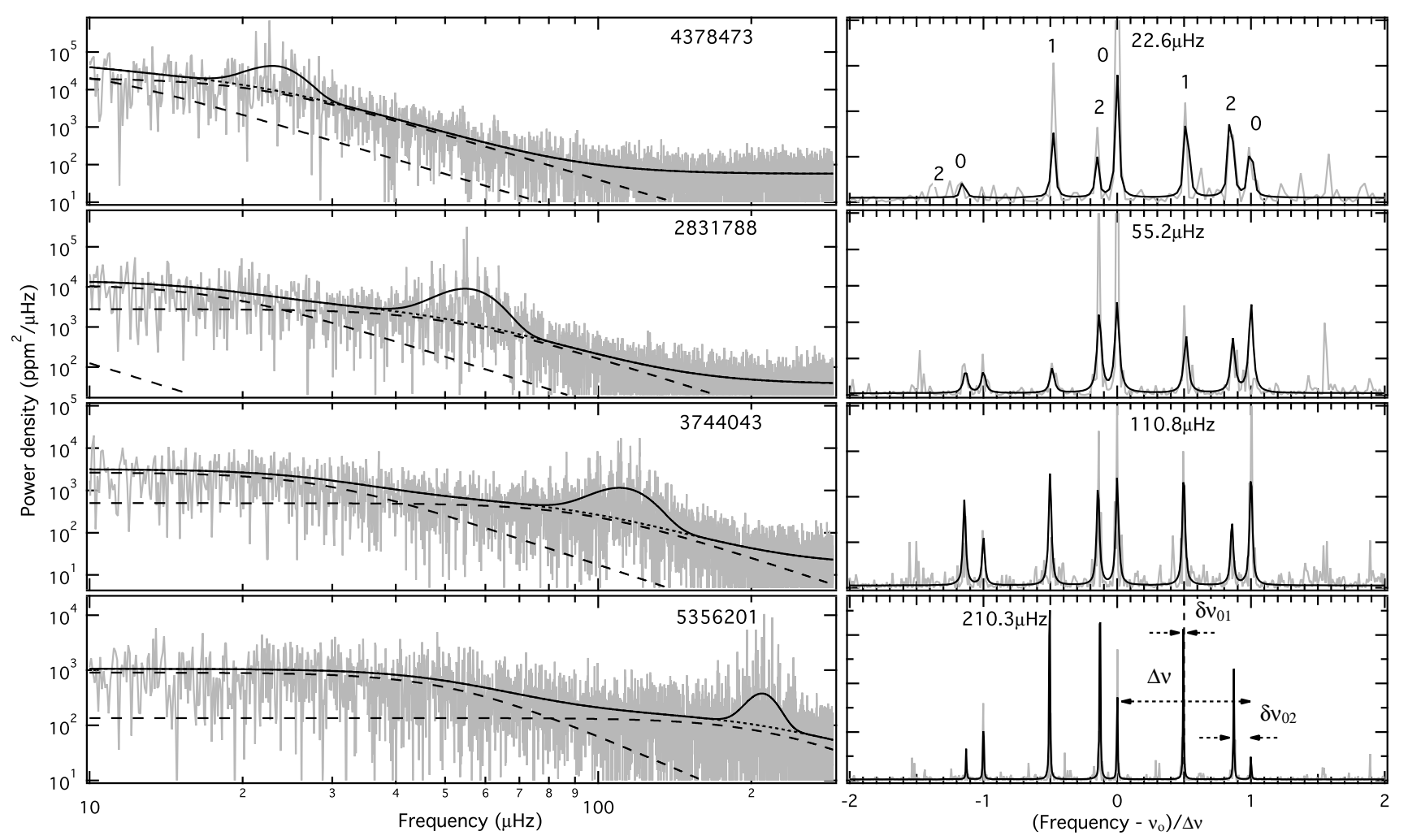

Fig. 2. Left panel: power density spectra for a sample of red giants observed with Kepler. Black lines indicate the global model fit and dotted lines show the global model plotted without the Gaussian component, which serve as a model for the background signal. Dashed lines indicate the background components. KIC numbers are given in the upper right corners. Right panel: residual power density spectra shifted to the central frequency (given in absolute numbers in the plots) of our model to determine the frequency separations and normalised to the large frequency separation. Black lines correspond to the best fitting model. The dashed line marks the midpoint between adjacent $l=0$ modes.

of the component is equal to half its value at zero frequency and is called the characteristic frequency. $P_{\mathrm{g}}, v_{\max }$, and $\sigma_{g}$ are the height, the central frequency, and the width of the power excess hump, respectively. Note that $\sigma_{g}$ is about 1.18 times the HWHM. For our sample of red giants, the frequency coverage of the Kepler observations allowed us to model up to three background components.

The only difference compared to the model in Paper I is the numerator in the background models. Originally, a single parameter, $A$, was used which corresponds to the power at frequency equal to zero of the given component. However, tests have shown that fitting $a$ and $b$ instead of fitting $A$ and $b$, with $A=2 \pi a^{2} / b$, yields a more robust fit and allows a more accurate measurement of the characteristic frequencies. This notation also makes more sense physically because $a^{2}$ corresponds to the variance that the signal produces in the time domain and can easily be related to the observed total energy of, e.g., granulation.

We used a Bayesian Markov-Chain Monte-Carlo (MCMC) algorithm to fit the global model to the power density spectra. See Paper I and Gruberbauer et al. (2009) for a detailed description. Briefly, the algorithm automatically samples a wide parameter space and delivers probability density distributions for all fitted parameters and their marginal distributions, from which we computed the most probable values and their $1 \sigma$ uncertainties. For the parameter limits, we followed a slightly modified approach to that in Paper I. During the fitting process we kept $v_{\max }$ within $\pm 25 \%$ of the value inferred from the visual inspection of the spectrum. The width of the power excess was allowed to vary between $5 \%$ and $50 \%$ of the initial guess of $v_{\max }$, where the lower limit prevented the algorithm from fitting the Gaussian to a single frequency bin in the spectrum. The frequency parameters $b_{i}$ were allowed to vary from 0 to 1.5 times $v_{\max }$, with the condition $b_{1}>b_{2}>b_{3}$, where the indices indicate consecutive background components. The amplitude parameters $a_{i}$ were kept between 0 and 10 times the square root of the highest peak power in the spectra. $P_{g}$ was allowed to vary from zero to 10 times the average power in the spectrum around the initial guess for $v_{\max }$, and $P_{n}$ was kept between 0.5 and 2 times the average power at the high frequency end of the spectrum. The left panel of Fig. 2 shows examples of power density spectra with the corresponding fits.

An important aspect of our analysis is to understand the uncertainties of the determined parameters. One might expect that the white noise, and therefore the brightness of a star, is responsible for a significant part of the uncertainty in $v_{\max }$ but we do not find any correlation with the magnitude. On the other hand, there is a clear correlation between $\sigma_{v \max }$ and the ratio of the height-to-background ratio (HBR) to the width of the power excess $\left(\sigma_{g}\right)$, where we define the HBR as the ratio between the height of the power excess $\left(P_{g}\right)$ and the background signal at $v_{\max }$. In other words, we can more accurately determine the centre of a narrow power excess hump with a large HBR than the centre of a broad hump with a small HBR. This is illustrated in Fig. 3 where we plot the absolute value of $\sigma_{v \max }$ (right axis) as a function of HBR $/ \sigma_{g}$. Tests with subsets of the Kepler time series (and data sets from CoRoT) have shown that $\sigma_{v \max }$ is also directly proportional to the frequency resolution of the time series, which is defined as the inverse data set length. To account for 


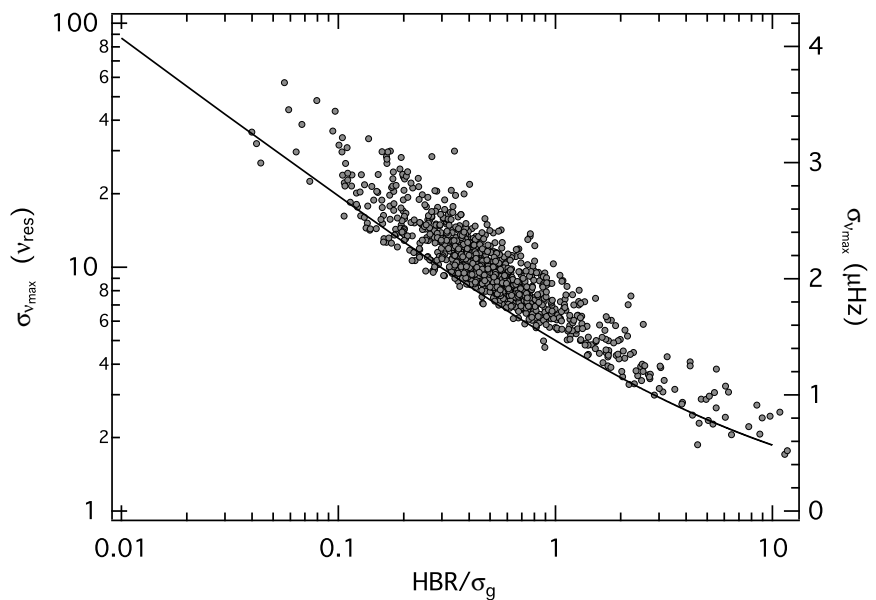

Fig. 3. The uncertainty in $v_{\max }$ in actual value (right axis) and in units of the frequency resolution as a function of the ratio between the heightto-background ratio (HBR) and the width of the power excess, $\sigma_{g}$. The line indicates a power-law model for the lower envelope.

this we plot $\sigma_{v \max }$ in units of the frequency resolution $\left(v_{\text {res }}\right)$ on the left axis in Fig. 3. From that, we can define a simple relation for the lower limit of the uncertainty in $v_{\max }$ as

$\sigma_{v \max }=v_{\text {res }}\left(1+\frac{4}{\left(H B R / \sigma_{g}\right)^{2 / 3}}\right)$.

For the solar case we would expect an uncertainty in $v_{\max }$ of about $5.3 \mu \mathrm{Hz}$, which is in good agreement with the value of $5.23 \mu \mathrm{Hz}$ found in Sect. 3.3 for SOHO/VIRGO data. Given this relatively simple error law we are confident that our uncertainties for $v_{\max }$ are reliable and do mainly reflect the constraints of the observations and not of our method. It is also interesting to see that the uncertainty of $v_{\max }$ is very much defined by the star itself, since $\mathrm{HBR} / \sigma_{g}$ is largely intrinsic to the star, for a given instrument and observing time. Nevertheless, we mention that the error law is purely phenomenological and might not be valid outside the range we use it.

A histogram of the relative uncertainties in $v_{\max }$ is given in the top panel of Fig. 4, showing a clear peak at about $2 \%$. This is not surprising as a large fraction of the analysed red giants are red-clump stars having a very similar $v_{\max }$, and therefore a similar width and height-to-background ratio of the power excess. We see that, for almost all stars, we could determine $v_{\max }$ to within $4 \%$ and, for about half of our sample, to within $2 \%$.

A potential problem for the subsequent analysis is that we assumed the power excess hump to be symmetric, so that an intrinsic asymmetry might result in a systematic error. In Paper I it was claimed that the asymmetry of the power excess hump is within the observational uncertainties of $v_{\max }$, and therefore negligible. However, that conclusion was based on the analysis of only 31 stars. Our sample is more than 30 times larger and should give a statistically more significant conclusion. We computed the weighted mean frequency, $v_{\mathrm{wm}}$, in the frequency range of pulsation $\left(v_{\max } \pm 3 \sigma_{g}\right)$, where we used the residual power after correcting for the background signal as weight. We found $v_{\mathrm{wm}}$ consistently shifted towards higher frequencies compared to $v_{\max }$ by $3.1 \pm 1.3 \%$ and therefore outside the average uncertainty for $v_{\max }$ of about $2.3 \%$. This is, however, not a problem in our subsequent analysis since we find the same shift of about $3 \%$ in the solar data (see Sect. 3.3), and as long as we compare $v_{\max }$ values that are determined in the same way, we do not have to take into account asymmetries in the power excess humps.

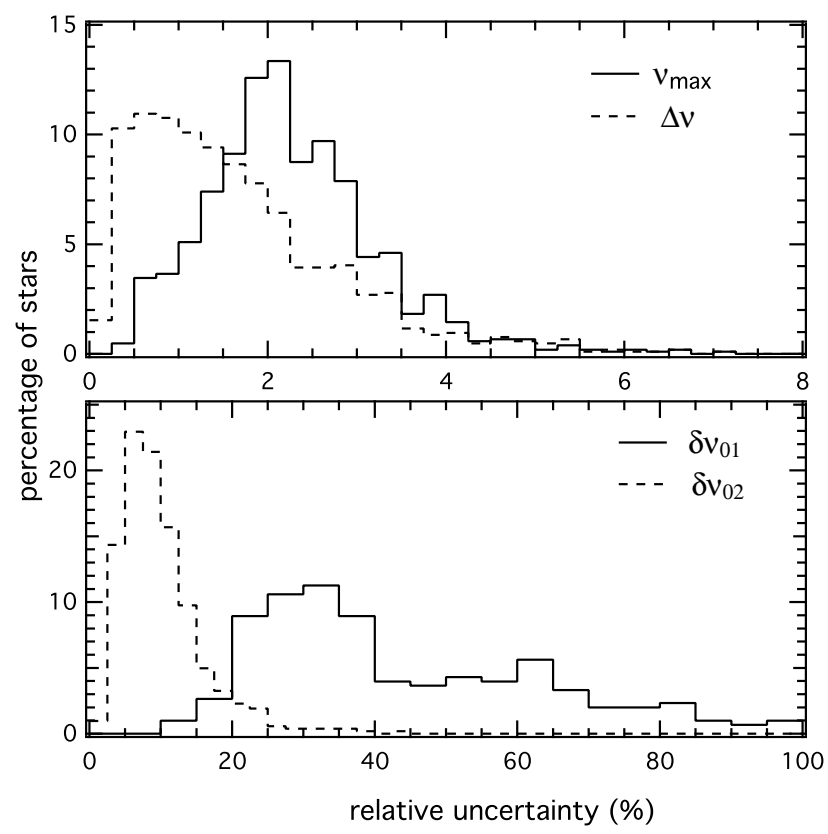

Fig. 4. Histograms of the relative uncertainties for $v_{\max }$, the large frequency separation $\Delta v$, and the small separations $\delta v_{01}$ and $\delta v_{02}$.

\subsection{Frequency spacings}

In the next step we used the white noise and background components of the global model (dotted lines in Fig. 2) to correct the power density spectra for the background signal, leaving only the oscillation signal and white noise. The second parameter that can directly be determined from the observed power spectrum is the large frequency separation, $\Delta v$. To determine $\Delta v$, we use a similar approach as in Paper I and fit the following general model to the residual power density spectrum over a frequency range spanning three radial orders around the frequency of maximum oscillation power:

$$
\begin{aligned}
P(v)= & P_{n}+\sum_{i=-1}^{1} \frac{A_{i}^{2} \tau}{1+4\left[v-\left(v_{0}+i \Delta v\right)\right]^{2}(\pi \tau)^{2}} \\
& +\sum_{j=-1}^{1} \frac{A_{j}^{2} \tau}{1+4\left[v-\left(v_{0}+j \Delta v-\delta v_{02}\right)\right]^{2}(\pi \tau)^{2}} \\
& +\sum_{k=-1,1} \frac{A_{k}^{2} \tau}{1+4\left[v-\left(v_{0}+\frac{k}{2} \Delta v+\delta v_{01}\right)\right]^{2}(\pi \tau)^{2}}
\end{aligned}
$$

The model represents a sequence of eight Lorentzian profiles whose frequencies are parameterised by a central frequency, $v_{0}$, and three spacings, $\Delta v, \delta v_{01}$, and $\delta v_{02}$, where the first, second, and third sum corresponds to three radial, three $l=2$, and two $l=1$ modes, respectively. $A_{i}, A_{j}$, and $A_{k}$ are the individual $\mathrm{rms}$ amplitudes. As in Paper I, we assume the same mode lifetime $\tau$ for all modes, which might not be true in reality, but this assumption has no impact on the determination of the spacings and significantly stabilises the fit. We again used the Bayesian MCMC algorithm to fit the model to the residual power density spectrum. All mode amplitudes were allowed to vary independently between zero and 10 times the highest peak in the amplitude spectrum. This allowed the algorithm to account for missing modes or modes hidden in the noise. The mode lifetime was sampled between 1 and 100 days. Most important here were the parameter ranges for the spacings. With the condition 
that $\delta v_{01} \ll \Delta v$ and $\delta v_{02}<\Delta v / 2$, the model basically represents the asymptotic relation (Tassoul 1980) for low-degree and highradial order $\mathrm{p}$ modes. Consequently, $\delta v_{02}$ and $\delta v_{01}$ correspond to the small separations between adjacent $l=0$ and 2 modes and between $l=1$ modes and the midpoint of consecutive radial modes, respectively. A critical parameter is the central frequency because the central mode must be a radial mode. Otherwise, the interpretation of the spacings contradicts the asymptotic relation. It turned out when allowing $v_{o}$ and $\Delta v$ to vary over a relatively wide range ( $\nu_{\max } \pm 2 \sigma_{g}$ for $v_{o}$ and $0.5 \mu \mathrm{Hz}$ to $2 \sigma_{g}$ for $\Delta v$ ) the algorithm was able to automatically find the central mode that corresponds to a radial mode if $l=2$ modes were present. In case of no detectable $l=2$ modes, the mode identification is ambiguous, but the delivered large spacing was still a good estimate.

We have determined $\Delta v$ for all 1041 stars and found at least two $l=2$ modes in the oscillation spectrum of about half of our sample, which allowed us to determine small spacings based on an automated mode identification. In principle, we have also $\delta v_{01}$ values for these stars but only accepted them for about one third of the total sample. This is because of the multiple dipole modes over a relatively broad frequency range per order, which occur due to their mixed gravity/acoustic mode character (Dupret et al. 2009), and makes it difficult for our algorithm to obtain robust results. We do not further investigate the small spacings here but refer to Huber et al. (2010), where those results are presented.

The oscillation spectra and the corresponding best fits for the stars in Fig. 2 are illustrated in the right panel of that figure. As for our global fit parameters, we determined the most probable parameters from the marginal distributions of the probability density delivered by the MCMC algorithm. Unlike for $v_{\max }$, we were not able to find a clear correlation between the uncertainty in $\Delta v$ and any other parameter combination. We expect, however, that $\sigma_{\Delta v}$ depends on the frequency resolution, the signal-tonoise ratio of the individual modes and their lifetime. We could not reliably determine the mode lifetimes for many stars because their mode profiles are undersampled, which means that the peak width due to the spectral window function of the observations is broader than the actual profile width. In such a case our amplitudes and lifetimes are meaningless. The mode frequencies and therefore the spacings are, however, not affected by this phenomenon. Histograms for the uncertainties of the spacing parameters are given in Fig. 4, showing that we can determine $\Delta v$ to within $1 \%$ for about $30 \%$ of our sample. Whereas the accuracy of $\delta v_{02}$ is mostly better than $10 \%$, the relative errors for $\delta v_{01}$ are relatively large. But one has to keep in mind that $\delta v_{01}$ is well below $1 \mu \mathrm{Hz}$ for a red giant and the absolute uncertainties of $\delta v_{01}$ are still quite low.

Originally, we would have had to exclude a number of stars from our sample because they pulsate with low frequencies making it difficult to reliably determine spacings from a frequency spectrum. There is, however, the so-called autocorrelation method described by Mosser \& Appourchaux (2009), which measures $\Delta v$ from the first peak in the autocorrelation of the time series. This method is less affected by the limited duration of the observations than Fourier-based methods and is able to detect regular spacings down to a few times the frequency resolution (Mosser et al. 2010). To account for this, we used the $\Delta v$ values from the autocorrelation method for all stars with $v_{\max }<15 \mu \mathrm{Hz}$ ( $\sim 7 \%$ of the total sample) for the subsequent analysis.

Finally, we cross-checked our results for $v_{\max }$ and $\Delta v$ with those of other methods (Hekker et al. 2010a; Huber et al. 2009; Mathur et al. 2010; Mosser \& Appourchaux 2009) which have been used to determine the same seismic parameters for our sample (or subsample) of red giants. A direct comparison of the different methods shows that there are a number of outliers but for most stars in our sample, at least one other method gave a value that is compatible with our results (i.e., within the uncertainties). Additionally, we have carefully checked by hand the reliability of the seismic parameters for all stars for which we found a significant disagreement in the direct comparison (see Hekker et al. 2010c, for the detailed comparison) and identified only a few stars (less than 1\%) which we had to eliminate from our sample because their seismic parameters are ambiguous.

\subsection{Solar reference parameters}

The large frequency separation is related to the inverse sound travel time through the star and therefore to the mean density of the star. It scales as $\Delta v_{\odot}\left(M / R^{3}\right)^{1 / 2}$ from the solar case, with $R$ and $M$ being the total mass and the radius of the star, respectively, in solar units. An important point when using scaling relations to estimate fundamental parameters is the definitions of the scaled seismic parameters. An often used solar reference value, $\Delta v=$ $134.95 \mu \mathrm{Hz}$ is based on the frequency difference between the radial modes with order $n=20$ and 21 where the maximum oscillation power is seen (Toutain \& Froehlich 1992). The frequency difference of two single modes is difficult to determine for other stars and an average value of all (or some) observable modes is often used (e.g., $\sim 134.8 \mu \mathrm{Hz}$ for the Sun; Kjeldsen et al. 2008). However, the frequency separation is a function of the frequency itself (see e.g. Broomhall et al. 2009 for the Sun and Mosser et al. 2010 for CoRoT red giants), and an average $\Delta v$ will depend on the actual number and frequency range of the observed modes and is difficult to compare for different observations.

The frequency of maximum oscillation power, on the other hand, is related to the acoustic cut-off frequency in the stellar atmosphere (e.g., Brown et al. 1991; Kjeldsen \& Bedding 1995), which in turn scales as $v_{\max , \odot}\left(M R^{-2} T_{\mathrm{eff}}^{-1 / 2}\right)$ from the solar case, where often used values are $v_{\max , \odot}=3050 \mu \mathrm{Hz}$ (e.g., Kjeldsen \& Bedding 1995) or $3100 \mu \mathrm{Hz}$ (e.g., Basu et al. 2010). Both seismic parameters scale with the stellar mass and radius and can therefore be used to estimate $R$ and $M$ of a star from its seismic parameters. But to use these seismic scaling relations in a consistent way we need values for the solar parameters, measured in the same way as for our sample of red giants. Note that the seismic scaling relations are not laws of physics and possibly include some additional uncertainties. There are, however, strong indications that at least the $\Delta v$-scaling is quite accurate for cool stars like our sample of red giants (Basu et al. 2010; Stello et al. 2009a).

We used a 1-year time series from the green channel of the SOHO/VIRGO data (Frohlich et al. 1997) obtained during a solar activity minimum and fitted our global model (Eq. (1)) to the corresponding power density spectrum. The result is given in Fig. 5, showing the original power density spectrum and a smoothed version of the average power density spectrum of nine consecutive 1-year time series along with the best fitting model. Although the model is fitted to "only" a 1-year time series, it almost perfectly reproduces the average spectrum of the full 9year time series. The reason why we do not use the 9-year time series is because of limited computer resources. Also shown is the probability density function of the solar $\nu_{\max }$ parameter, from which we determined $\nu_{\max , \odot}=3120 \pm 5 \mu \mathrm{Hz}$.

In the next step we use the background part of the global model fit to correct the power density spectrum and fit the asymptotic relation model to the residual spectrum. The solar 


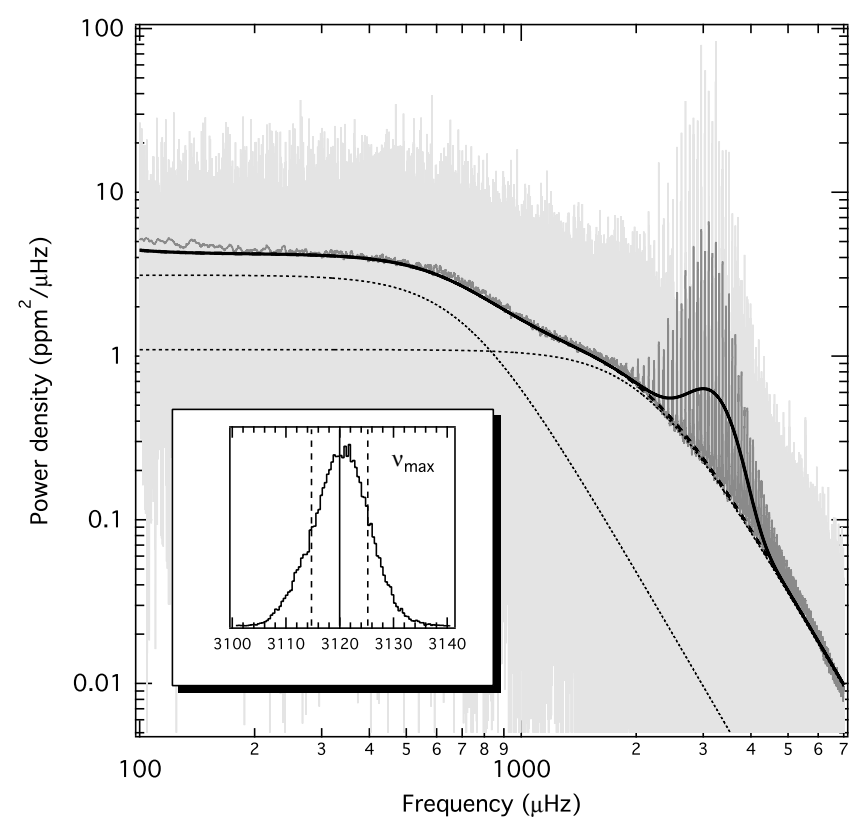

Fig. 5. Power density spectrum of a 1-year VIRGO time series (lightgrey) and the corresponding global model fit (black line). The dark-grey line shows a smoothed ( $5 \mu \mathrm{Hz}$ boxcar filter) version from the average of nine consecutive 1-year VIRGO time series. The inset gives the probability density function for $v_{\max }$ with the vertical solid and dashed lines indicating the median value and the $1 \sigma$ limits, respectively.

p-mode profiles are naturally much better resolved than the pmodes in our sample of red giants and initial tests have shown that the rotationally split components of the non-radial modes significantly disturb the fit. To account for this we have included rotationally split components for the $l=1$ and 2 modes, which are parameterised by a single rotational frequency and an inclination angle that defines the height ratio between the central profile and the split components (e.g., Gizon \& Solanki 2003; Ballot et al. 2006). We have also tested asymmetric mode profiles (Nigam et al. 1998) but found no significant influence on the parameters of interest. In Fig. 6, we show the probability density distributions for the three frequency spacings and the central frequency of our model, together with their median values and $1 \sigma$ limits. The bottom panels illustrate the residual power density spectrum and the best model fit. We have determined $\Delta v_{\odot}=134.88 \pm 0.04 \mu \mathrm{Hz}, \delta v_{02, \odot}=9.00 \pm 0.06 \mu \mathrm{Hz}$, and $\delta v_{01, \odot}$ $=6.14 \pm 0.10 \mu \mathrm{Hz}$. The uncertainties might appear unrealistically small but they reflect a well defined case specifically chosen for our approach and should not be mistaken as "global" frequency spacings of the Sun.

\section{Asteroseismic fundamental parameters}

\subsection{Method}

Our approach to determining an asteroseismic mass and radius depends on the aforementioned scaling relations for $v_{\max }$ and $\Delta v$ :

$$
\begin{aligned}
v_{\max } & =3120 \pm 5 \mu \mathrm{Hz} \times M R^{-2} T^{-1 / 2} \\
\Delta v & =(134.88 \pm 0.04 \mu \mathrm{Hz}) \times M^{1 / 2} R^{-3 / 2}
\end{aligned}
$$

with $M$ and $R$ in solar units and $T=T_{\text {eff }} / 5777 \mathrm{~K}$. Obviously the $v_{\max }$ scaling relation depends on the effective temperature of the star. Although there are temperatures available in the KIC for most of the analysed stars, we decided not to directly use them in our analysis (i.e., in the above scaling relations). The KIC parameters $\left(T_{\text {eff }}, \log g\right.$, and $\left.[\mathrm{Fe} / \mathrm{H}]\right)$ are mostly determined from multi-colour photometry and they are calibrated to be correct in a statistical sense, i.e., the average values of a large sample of stars are correct. However, a star-by-star comparison (e.g., for stars associated in a cluster) has shown that the individual values can have a large scatter (up to some $100 \mathrm{~K}$ in $T_{\text {eff }}$ ). Consequently, adopting the KIC temperatures would add uncertainties of up to $5 \%$ and $10 \%$ to our radius and mass determinations, respectively. Therefore, we also compared the seismic parameters of the observed stars and their KIC temperatures to those inferred from stellar models, and then adopted the fundamental parameters from the model that best reproduced all input parameters. There are several ways to do this. In Paper I, an initial guess for the stellar mass and radius was determined using an average temperature for stars on the red giant branch. The initial mass and radius values were then compared to those of a grid of solar-calibrated red-giant models to get a better estimate for the temperature. The new temperature was adopted and the procedure repeated, converging to a certain location in the H-R diagram after a few iterations and delivering a full set of fundamental parameters for each star. However, these iteratively determined parameters also depend on the chemical composition and the evolutionary status of the model (i.e., whether the model is a red-giant branch or an asymptotic-giant branch model). This ambiguity allows a given set of seismic parameters to converge to different locations in the H-R diagram if different model grids with, e.g., a different chemical composition are used. This uncertainty has, however, only small effects on the mass and radius determination. For the effective temperature and luminosity it adds about $\pm 150 \mathrm{~K}$ and $\pm 20 \%$ uncertainties, respectively.

A more general approach was presented by Basu et al. (2010). They used a combination of seismic and conventional stellar parameters and compared them to those of a multi-metallicity model grid, where the aforementioned scaling relations are used to determine the seismic parameters for the models. Basu et al. (2010) defined a model likelihood from the difference between the model and input parameters and infer the stellar radius from the resulting likelihood function. From their tests with a number of artificial stars they concluded that it is very unlikely to get a reasonable estimate for a red giants' radius if no accurate temperature and parallax are available. This conclusion was, however, based on only a single red-giant test star that is located high up the red giant branch and therefore quite far away from where most of the observed red giants are expected (i.e. in the red clump). Additionally, they have assumed uncertainties for the seismic input parameters that are about ten times larger than what we have determined for our sample of red giants.

Here, we follow the approach of Basu et al. (2010) but formulate our search for a best set of fundamental parameters in a Bayesian sense. Given a set of seismic input parameters $v_{\text {max obs }}$ and $\Delta v_{\mathrm{obs}}$, and the Gaussian distribution of their uncertainties, $\sigma_{v \max }$ and $\sigma_{\Delta v}$, we define the likelihood that the seismic model parameters, $v_{\text {max } \text {,model }}$ and $\Delta v_{\text {model }}$, matches the observed ones as

$$
\begin{aligned}
& \mathcal{L}_{v_{\max }}=\frac{1}{\sqrt{2 \pi} \sigma_{v_{\max }}} \exp \left(\frac{-\left(v_{\text {max }, \text { obs }}-v_{\text {max }, \text { model }}\right)^{2}}{2 \sigma_{v_{\max }}^{2}}\right) \\
& \mathcal{L}_{\Delta v}=\frac{1}{\sqrt{2 \pi} \sigma_{\Delta v}} \exp \left(\frac{-\left(\Delta v_{\text {obs }}-\Delta v_{\text {model }}\right)^{2}}{2 \sigma_{\Delta v}^{2}}\right)
\end{aligned}
$$



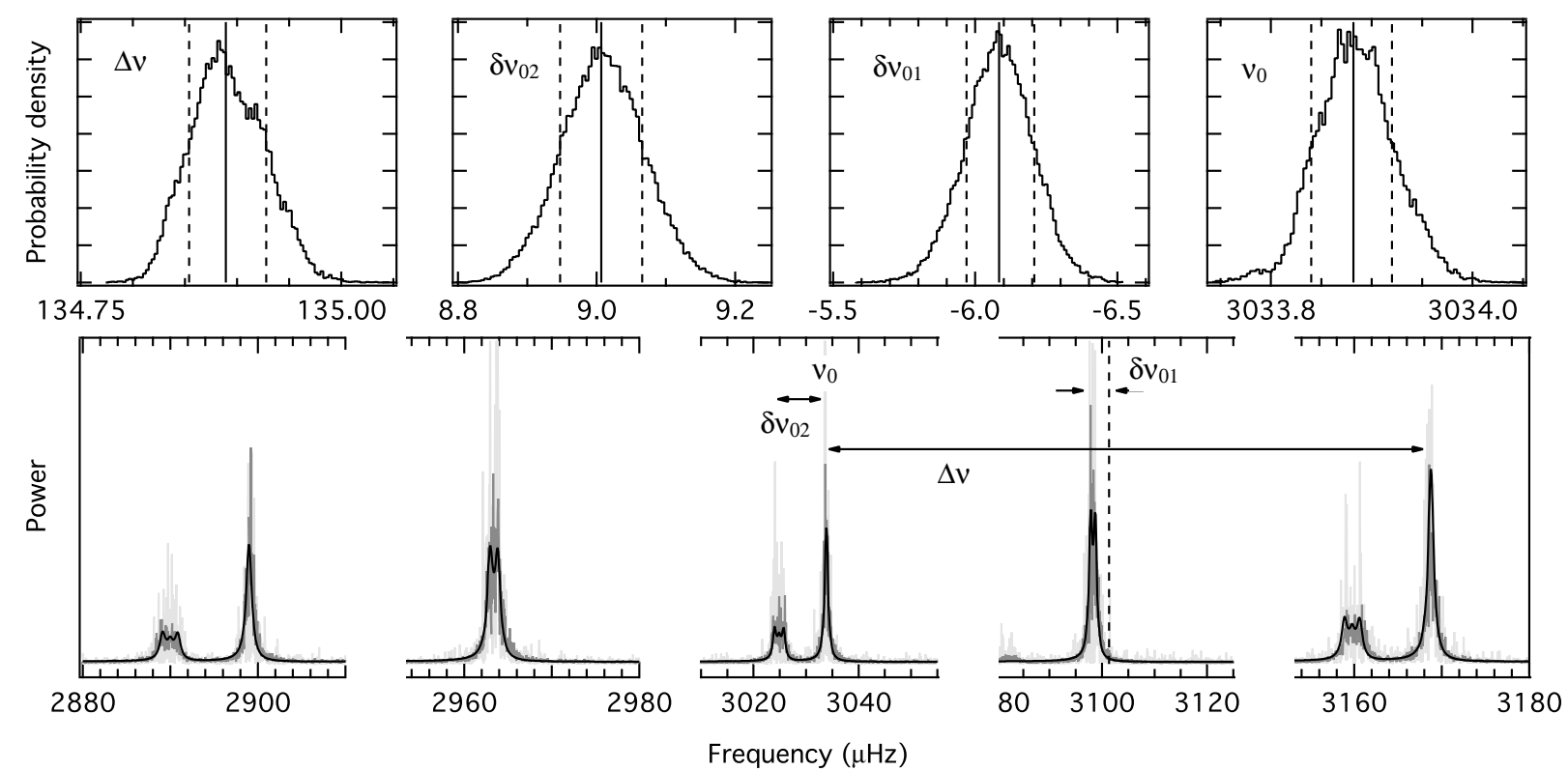

Fig. 6. Same as Fig. 5 but as delivered from our algorithm to determine the frequency spacings. Top panels: probability density functions for the three spacing parameters and the central frequency of our model fit. Median values and $1 \sigma$ limits are indicated by vertical solid and dashed line, respectively. Bottom panel: residual power density spectrum (light-grey) of a 1-year VIRGO time series and the corresponding model fit (black line).

To simplify matters, we also assume a Gaussian error for the KIC effective temperatures and define the likelihood that the model matches the observed temperature as

$\mathcal{L}_{T_{\text {eff }}}=\frac{1}{\sqrt{2 \pi} \sigma_{T_{\text {eff }}}} \exp \left(\frac{-\left(T_{\text {eff, } \mathrm{KIC}}-T_{\text {eff,model }}\right)^{2}}{2 \sigma_{T_{\text {eff }}}^{2}}\right)$,

with a typical value of $200 \mathrm{~K}$ for $\sigma_{T_{\text {eff }}}$. In the Bayesian approach we can assign an overall probability of the model $M_{i}$ with the posterior probability $I$ matching the observed parameters $D$ with respect to the entire set of models according to Bayes theorem as

$p\left(M_{i} \mid D, I\right)=\frac{p\left(M_{i} \mid I\right) p\left(D \mid M_{i}, I\right)}{p(D \mid I)}$

where

$p\left(M_{i} \mid I\right)=\frac{1}{N_{m}}$

is the uniform prior probability for a specific model with $N_{m}$ being the total number of models, and

$p\left(D \mid M_{i}, I\right)=\mathcal{L}\left(v_{\text {max }}, \Delta v, T_{\text {eff }}\right)=\mathcal{L}_{v_{\text {max }}} \mathcal{L}_{\Delta v} \mathcal{L}_{T_{\text {eff }}}$

is the likelihood function. The denominator of Eq. (9) is a normalisation factor for the specific model probability in the form of

$p(D \mid I)=\sum_{j=1}^{N_{m}} p\left(M_{j} \mid I\right) \cdot p\left(D \mid M_{j}, I\right)$.

Since the uniform priors are the same for all models they cancel in Eq. (9), which simplifies to

$p\left(M_{i} \mid D, I\right)=\frac{p\left(D \mid M_{i}, I\right)}{\sum_{j=1}^{N_{m}} p\left(D \mid M_{j}, I\right)}$.
The resulting model probability distribution automatically translates into most probable fundamental parameters and their uncertainties by constructing the marginal distribution for the corresponding parameter. The normalised probability of the most probable parameters is therefore a measure of how likely they are with respect to the other models of the specific grid. We stress that it does not tell us how probable the parameters are in an absolute sense, although formally it must be implicitly assumed than one of the tested models is actually true. The probability must be interpreted as being restricted to the space of the models being considered, and their associated physics. This means that as soon as additional models are added to the model space, the probabilities will change.

We also mention for completeness that since we use an uniform prior that rates each model with the same prior probability our Bayesian approach is actually not very different from the likelihood method of Basu et al. (2010). But as soon as we would add additional informations such as, e.g., an initial mass function, the advantages of the Bayesian technique would become obvious. But this is beyond the scope of the present paper and we leave it to future investigations.

\subsection{Models}

The red-giant models used for our analysis were extracted from the canonical scaled-solar $\mathrm{BaSTI}^{3}$ isochrones (Pietrinferni et al. 2004) in version 5.0.0 with a mass-loss parameter $\eta=0.2$. The grid includes models which were evolved from the zeroage main-sequence up to the tip of the red-giant branch (RGB), down to the He-core burning main sequence (red clump) and back up to the asymptotic giant branch (ABG) to an age of about 15 Gyr. We restricted the grid to models that have already left the main sequence with initial chemical compositions of $(Z, Y)=(0.008,0.256),(0.01,0.259),(0.0198,0.2734),(0.03$,

\footnotetext{
3 Available from http://albione.oa-teramo.inaf.it/
} 
$0.288)$, and $(0.04,0.303)$. The model mass ranges from 0.7 to $4 M_{\odot}$ with steps of typically $0.02 M_{\odot}$. We rejected models both with a mass above $4 M_{\odot}$ and with metallicities below $Z=0.008$ because they develop a distinctive horizontal giant branch that would significantly complicate our analysis.

We think this is justified because very high mass and very metal-poor stars are both quite rare and it is unlikely to find more than a few in a random sample of red giants. Initial tests showed that the poor resolution in chemical composition leads to an artificial clustering of stars in the H-R diagram, i.e., the redclump stars are concentrated in sharp features corresponding to the red-clump models of the different metallicity grids. We therefore increased the resolution in chemical composition to steps of 0.002 in $Z$ via interpolating the effective temperature for models of approximately constant mass and radius but with different chemical composition. The corresponding luminosities were determined from the Stefan-Boltzmann law, $L \propto R^{2} T^{4}$. The final grid used consists of about 1.4 million models based on about 340000 original BaSTI models.

\subsection{Testing the method}

To test our algorithm we use test star 6 from Basu et al. (2010) from their Table 1. The input $v_{\max }$ and $\Delta v$ are $21 \pm 0.2$ and $2.2 \pm 0.05 \mu \mathrm{Hz}$, respectively, where we adopt typical uncertainties from our sample of red giants. The resulting model probabilities and cumulative probability distributions for the model radius are illustrated in Fig. 7, from which we infer the most probable radius and its uncertainty to be $R=22.1 \pm 0.9 R_{\odot}$, which is in good agreement with the input radius of $21.44 R_{\odot}$ (Basu et al. 2010).

A more realistic test case is the red giant $\epsilon \mathrm{Oph}$, for which an interferometric radius is available. With $v_{\max }=53.5 \pm 2.0, \Delta v=$ $5.2 \pm 0.1 \mu \mathrm{Hz}$ (Kallinger et al. 2008a), and a spectroscopic $T_{\text {eff }}=$ $4877 \pm 100 \mathrm{~K}$ (De Ridder et al. 2006) we determined a radius of $10.7 \pm 0.4 R_{\odot}$, which is in good agreement with the interferometric radius of $10.55 \pm 0.15 R_{\odot}$ (Mazumdar et al. 2009). The other parameters are also in fairly good agreement with independent measurements. Our mass estimate of $1.89 \pm 0.08 M_{\odot}$ compares well to stellar masses determined from a detailed modelling: $1.85 \pm 0.05 M_{\odot}$ (Mazumdar et al. 2009) and 2.02 $M_{\odot}$ (Kallinger et al. 2008a). Even our luminosity estimate of $59 \pm 5 L_{\odot}$ is compatible with the luminosity of $58 \pm 4 L_{\odot}$ based on the Hipparcos parallax (van Leeuwen 2007).

\subsection{Results for Kepler stars}

In a first step, we have excluded 65 red giants which are associated with clusters from our sample because they might bias the subsequent analysis. Figure 8 shows the radius as a function of the mass for the remaining sample of red giants. Whereas in the top panel, $R$ and $M$ are directly determined from the seismic scaling relations adopting the KIC temperatures, the bottom panel shows $R$ and $M$ as they follow from the Bayesian comparison with the stellar model grid. Both distributions include many stars located in a narrow range around ten solar radii. Most of the stars in this range are expected to correspond to the red clump (e.g., Miglio et al. 2009) and their large number is due to the different evolutionary rates of giant-branch stars. Whereas stellar evolution takes place quite rapidly during the RGB phase and after the He flash at the tip of the RGB, it significantly slows down during the quiescent He-core burning phase in the red clump. Hence one can expect to find more red-clump stars and therefore
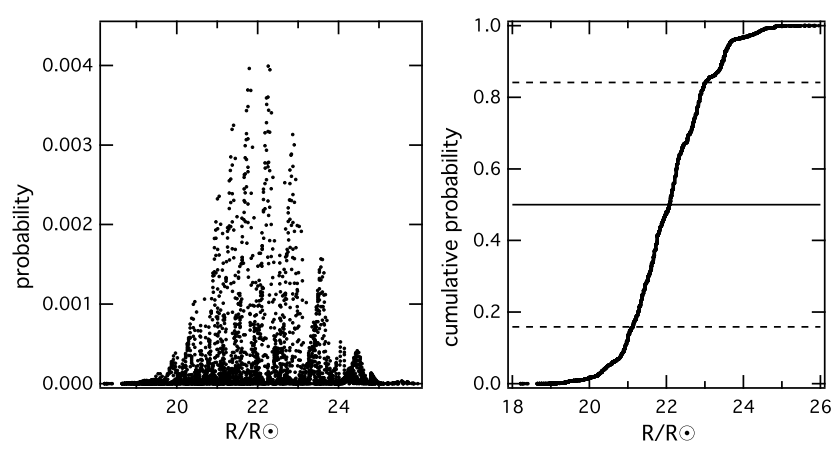

Fig. 7. The projected probability (left) and cumulative probability (right) distribution functions for the radius of the artificial test star 6 (Basu et al. 2010). The full horizontal line correspond to the median and the dashed lines give the $\pm 1 \sigma$ confidence interval.

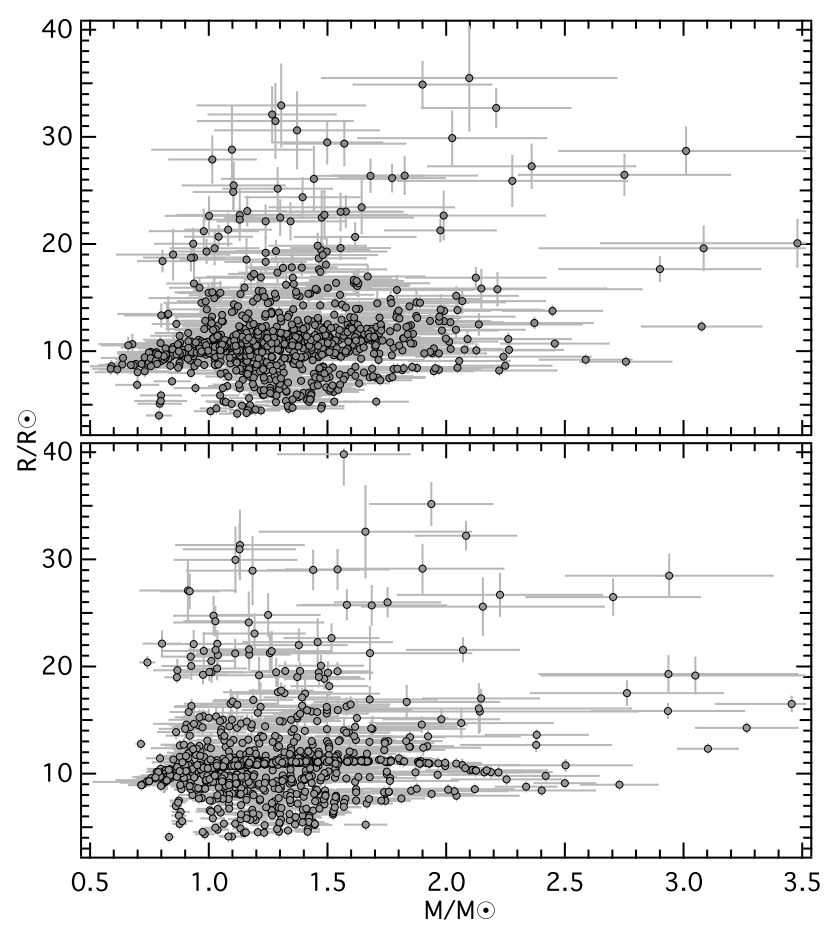

Fig. 8. The stellar radius as a function of mass for the sample of red giants as directly determined from Eqs. (4) and (5) using the KIC effective temperatures (top) and from the comparison with the stellar model grid (bottom).

stars with a similar radius than RGB stars in a random sample of red giants.

Although the two methods to determine $R$ and $M$ give a similar picture there are some important differences. Firstly, the verylow-mass stars in the top panel are most likely artefacts because, apart from binary stars which have lost a significant fraction of their mass, the universe is not old enough for 0.5-0.7 solar mass stars to have become 10-15 solar radii giants. Secondly, the distribution of stars below the red clump seems to be more realistic in the bottom panel than in the top panel. The higher mass stars evolve faster than the lower mass stars and therefore fewer higher mass stars should be present in a random sample of giants. Additionally, the error bars are in general smaller in the bottom panel and the red-clump feature is more pronounced. We are therefore confident that the additional efforts in determining the fundamental parameters are justified because they enable a more detailed picture of the stellar population on the giant branch. 


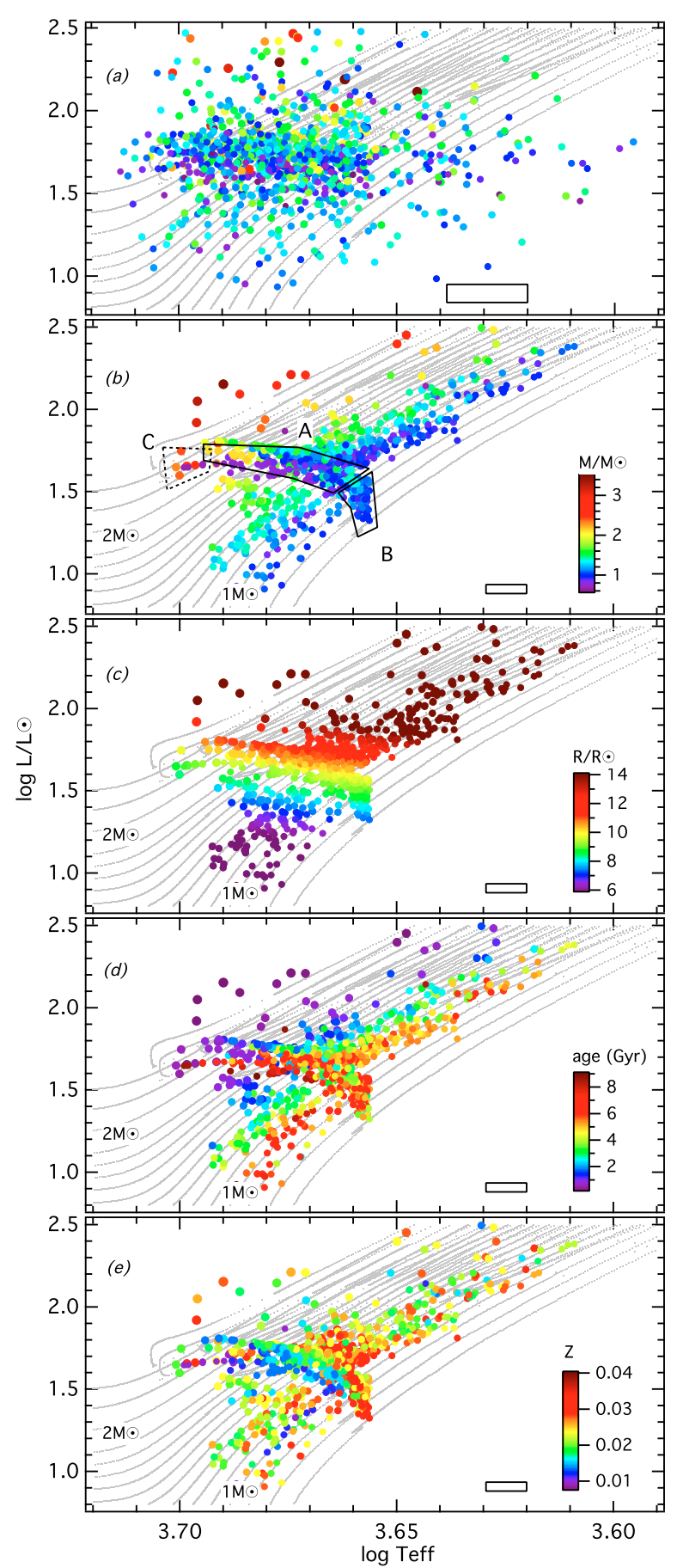

Fig. 9. H-R diagram showing the location of our red-giant sample as directly determined from Eqs. (4) and (5) using the KIC effective temperatures (panel a)) and from the comparison with the stellar model grid (panel b) to e)). The colour code (in the online version only) indicates the stellar mass (a) and b)), radius c)), age d)), and metallicity (e)), where the radius scale has been truncated below 6 and above $14 R_{\odot}$. Grey lines show solar-metallicity BaSTI evolutionary tracks. The boxes given in the lower right corners illustrate typical uncertainties.

\subsubsection{Kepler red giants in the $\mathrm{H}-\mathrm{R}$ diagram}

As our algorithm also delivers effective temperatures and luminosities we can put the analysed stars in a H-R diagram. Panel a of Fig. 9 shows the model-independent positions in the H-R diagram, with the temperatures from the KIC and the luminosities following from the Stefan-Boltzmann law, with $R$ directly determined from the seismic scaling relations (by using the KIC temperatures). The other panels show the positions in the H-R diagram as they follow from the Bayesian comparison with the stellar model grid with the colour code indicating the mass, radius, age, and metallicity of the best fit models. The distribution of stars in the H-R diagram reveals some interesting features. The most distinctive one (denoted as "A" in panel b of Fig. 9) consists of a large number of stars ( $35 \%$ of the total sample) lining up at almost constant luminosity and corresponds to the red clump. A similar feature (B) is located at slightly lower luminosities and with an almost constant temperature. It most likely corresponds to the so-called red bump, which is another characteristics of stellar evolution on the giant branch due to the outward-moving hydrogen-burning shell that burns through the mean molecular weight discontinuity left by the first dredgeup from the convective envelope causing a slight contraction of the star before the star starts to ascend the giant branch again. Significantly less populated than the red clump and bump is the feature (C) that corresponds to the so-called secondary clump (Girardi 1999) including high-mass stars $\left(>2 M_{\odot}\right)$ that settle as He-core burning stars at lower luminosities than the lower-mass red-clump stars. The secondary-clump population is of particular interest because its mass puts tight constraints on, e.g., the convective-core overshooting or the recent history of star formation in the Galaxy (Girardi 1999). We refer to Huber et al. (2010) who report on the signature of secondary-clump stars in the distributions of the seismic observables of red giants in the Kepler field of view (see also Mosser et al. 2010, for CoRoT red giants).

Combining the mass distribution in the H-R diagram (panel b in Fig. 9) with the radius distribution (panel c in Fig. 9) we infer that the red clump consists of about 0.8 to $1.8 M_{\odot}$ stars (with the low-mass stars accumulated at the bottom of the red clump) with a radius between about 10 to $12 R_{\odot}$. The red bump, on the other hand, is dominated by $1 M_{\odot}$ stars but with a lower average radius than the red-clump stars. The secondary-clump covers a similar radius range but includes stars with masses above about $2 M_{\odot}$. Outside the red clumps and bump, the stars follow the usual mass distribution with increasing masses towards higher temperatures and luminosities. The detailed structure in the H-R diagram is almost not visible in the model-independent approach (panel a). The uncertain KIC temperatures obviously blur the distribution of the stars in the red clumps and bumps. However, we note that the model-independent approach tentatively shows the mass gradient in the red clump as well.

Although we cannot constrain the metallicity to better than about $\pm 50 \%$ for individual stars, the metallicity distribution shows some interesting trends (see panel e in Fig. 9). In the red clump, there is a metallicity gradient ranging from metal-poor stars at the bottom to metal-rich stars at higher luminosities. And the red bump tentatively consists of metal-enhanced stars.

To analyse the red clump and bump in more detail we compare in Fig. 10 the observed features with different metallicity grids. As indicated in panel d of Fig. 9, the red clump is dominated by He-core burning stars of the solar-metallicity grid (middle panel of Fig. 10). The metallicity gradient in the red clump is due to the position of the He-core burning main-sequence in the different metallicity grids. Whereas the metal-poor models are shifted towards higher temperatures and therefore towards the bottom of the observed red clump, the metal-rich models accumulate at the opposite side of the red clump. Similar can be seen for the red bump. But there, metal-enhanced models are more consistent with the observed sample than solar-metallicity models. 


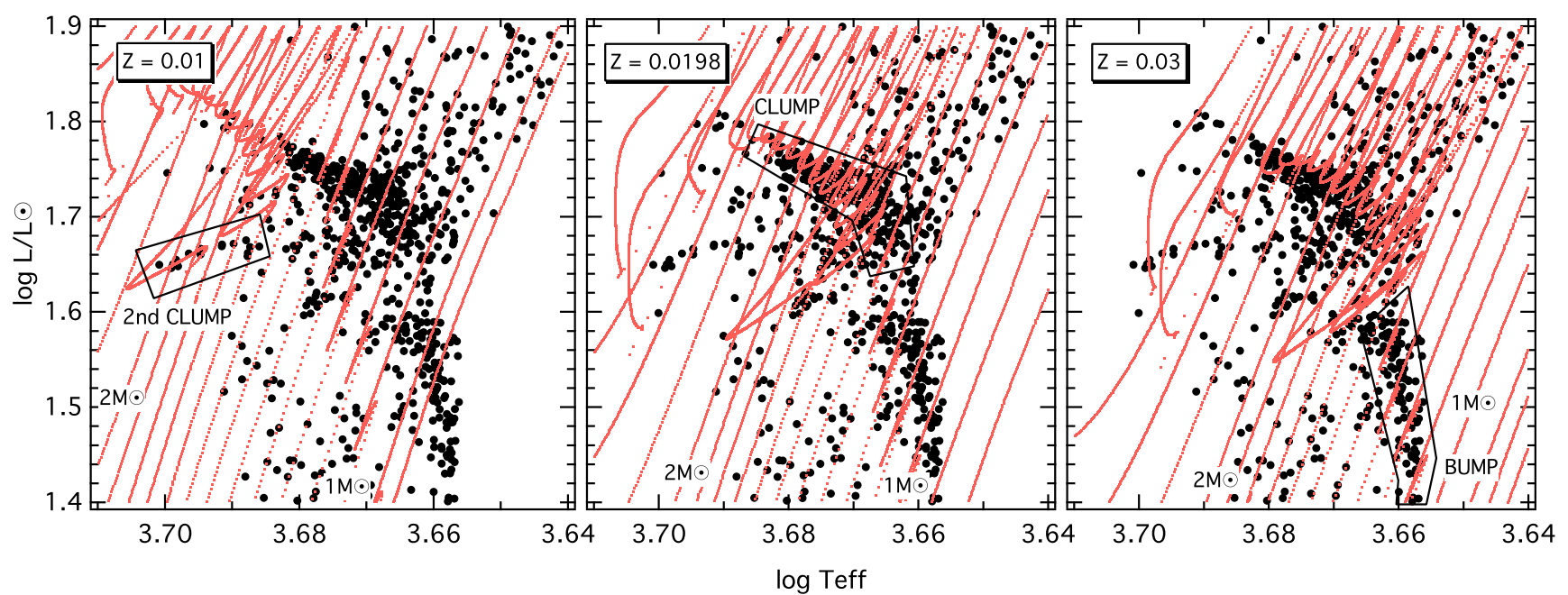

Fig. 10. H-R diagrams showing our sample of red giants (black dots) with respect to different metallicity model grids (red dots - in the online version only).

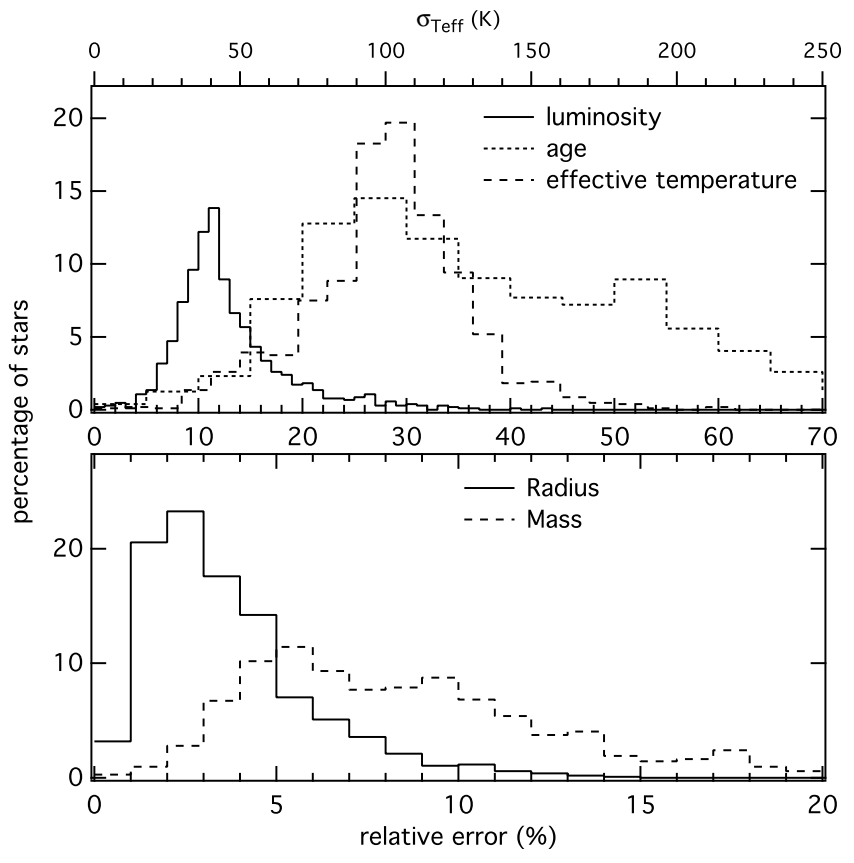

Fig. 11. Histograms of the relative (and absolute for $T_{\text {eff }}$ ) uncertainties for the asteroseismic fundamental parameter.

In Fig. 11 we show histograms for the relative uncertainties in mass and radius (bottom panel) showing that for about half of our sample we can constrain the mass and radius to within $7 \%$ and $3 \%$, respectively. These uncertainties are dominated by the observational uncertainties of the seismic input parameters and are only slightly affected by the model related parameters. On the other hand, the effective temperature and luminosity of a model with a given mass and radius can significantly differ for, e.g., a different initial chemical composition or mixing length parameter. Compared to Paper I, where only a single metallicity grid was used, we cover a wide range in chemical composition and therefore expect to get a more realistic uncertainty for the $\mathrm{H}-\mathrm{R}$ diagram position. Histograms for the relative uncertainties in effective temperature, age, and luminosity are given in the top panel of Fig. 11 showing that we can determine the effective temperature, age and luminosity to within $\pm 110 \mathrm{~K}, \pm 30 \%$, and $\pm 11 \%$, respectively, for most of the analysed red giants.

We have tested our results with the RADIUS (Stello et al. 2009b) and A2Z (Mathur et al. 2010) methods, which showed qualitative agreement. However, a direct comparison is meaningless since RADIUS and A2Z use only hydrogen burning models and therefore do not include the red clump.

\subsubsection{Radius and mass distributions}

The radius and mass distribution in our sample of red giants is given in Fig. 12. The radius distribution clearly shows two populations of stars that are located in the same region of the H$\mathrm{R}$ diagram. The $\mathrm{H}$-shell burning stars ascending the giant branch and the He-core burning stars in the red clump. The very different rate at which they change their fundamental parameters (e.g., the radius) results in two superposed components of their radius distribution. The main component is a broad Gaussianlike distribution with a maximum number of stars between 9.5 and $10 R_{\odot}$. This component is dominated by RGB stars ascending the giant branch and the maximum falls onto the average radius of the red bump (see panel c of Fig. 9) but also includes the secondary-clump stars. The RGB stars are superposed with the sharp distribution of red-clump stars with their radii ranging from 10.5 to $11.5 R_{\odot}$. Also interesting is the excess of stars with a radius around $20 R_{\odot}$. If real, this clustering of stars would be very interesting because it might indicate stars on the AGB whose He-burning shells burn through the discontinuity left by the second dredge up, which happens indeed at about 20 solar radii in solar metallicity models. But a significantly larger sample would be needed to verify if the excess is real.

The mass distributions in the bottom panel of Fig. 12 shows that there are only very few low-mass stars in our sample. Their number slowly increases between 0.8 to $1.5 M_{\odot}$ with a small excess between 1 to $1.2 M_{\odot}$, and rapidly drops for higher masses. To test for bias of our composite sample we computed the radius and mass distributions in the subsamples (red and blue bars in Fig. 12) and found no significant difference for the radius distribution. The mass distributions, on the other hand, are different. Obviously, there are more 1.3 to $1.5 M_{\odot}$ stars in the 


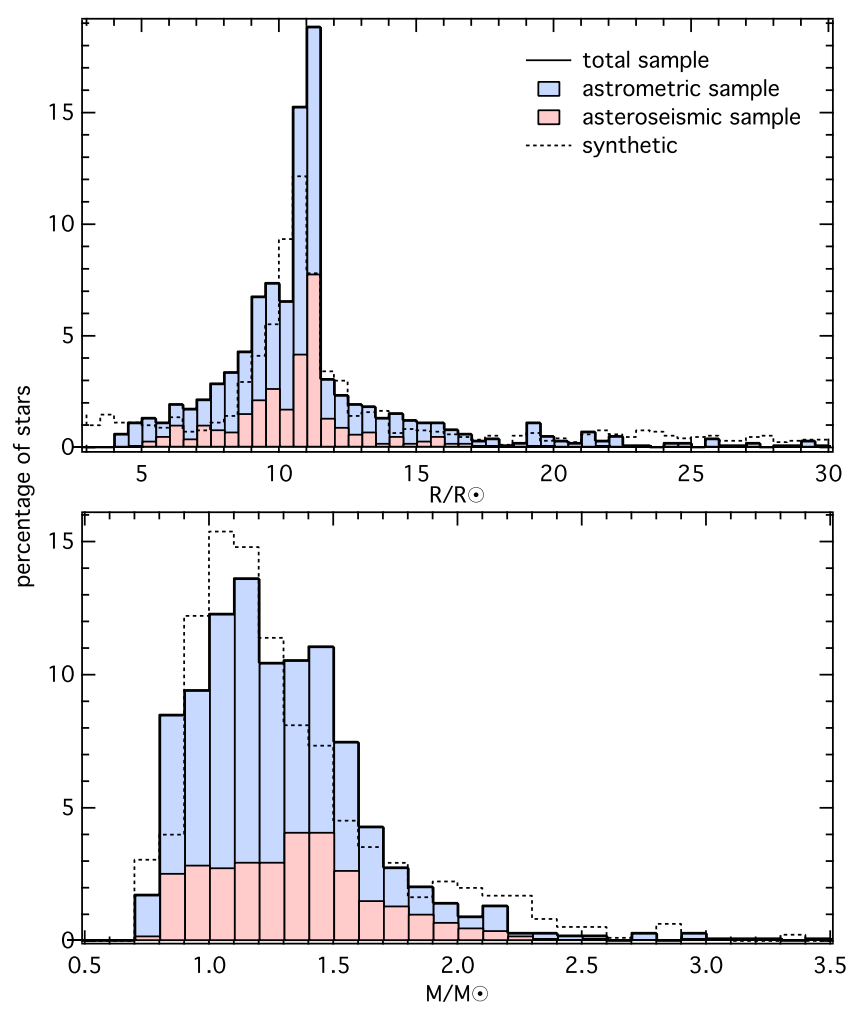

Fig. 12. Histograms for the radius and mass distribution in our sample of red-giant stars compared to the distribution of a synthetic red-giant population.

asteroseismic sample than in the astrometric sample showing its maximum between 1 to $1.2 M_{\odot}$. We expect the excess of "highmass" stars in the asteroseismic sample to be due to the original selection of the stars.

The detailed structure in the radius and mass distribution enabled us to identify different stellar populations in our sample which we can compare with synthetic populations for the Kepler field of view. To do so, we used the synthetic red-giant population for the Kepler field of view as presented by Huber et al. (2010) and computed with the stellar synthesis code TRILEGAL (Girardi et al. 2005) in the same way as by Miglio et al. (2009) for one of the CoRoT fields. The synthetic radius and mass distributions are indicated as dashed lines in Fig. 12. Although the comparison can not be done in an absolute sense as the observed sample is biased, the observed and synthetic distributions look quite similar but show also some interesting differences. The red-clump feature in the synthetic radius distribution is slightly broader and has its maximum at a lower radius compared to the observed distribution. Additionally, the RGB component is less pronounced, which is due to significantly less red-bump stars in the synthetic population. Since the stellar synthesis does not include stellar clusters, the main difference in the mass distributions is due to fewer 1.3 to $1.5 M_{\odot}$ stars in the synthetic distribution. More interesting is, however, the difference for highmass stars $\left(>2 M_{\odot}\right)$ indicating differently populated secondary clumps. Although these differences potentially carry detailed informations about the star formation history in the Kepler field of view and the associated physics, it would require detailed modelling to further investigate them, which is beyond the scope of this paper.
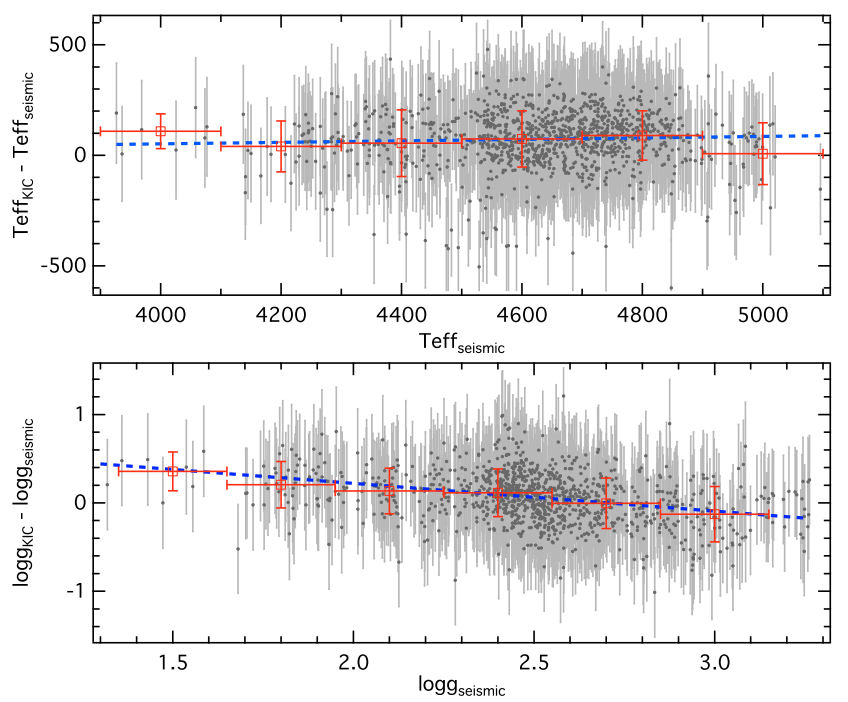

Fig. 13. Comparison between the seismically determined effective temperature and surface gravity and the corresponding KIC parameters. Dashed lines indicate a linear fit and red square symbols (in the online version only) show binned values.

\subsubsection{Testing the KIC parameters}

Finally, we compared in Fig. 13 (upper panel) the seismically determined effective temperature with the KIC temperature. We find the seismic temperature systematically shifted towards lower temperatures by about $50 \mathrm{~K}$ (see linear fit and binned values). The rms scatter (about $120 \mathrm{~K}$ ) of the temperature difference is, however, within the assumed uncertainties for the KIC temperatures of $200 \mathrm{~K}$ (priv. comm. Brown). The KIC uncertainties seem therefore to be overestimated but one has to keep in mind that our algorithm uses the KIC temperatures as an input parameter and the two temperature estimates are therefore not independent. A more meaningful indicator for the reliability of our fundamental parameter estimation is the surface gravity, which is also given in the KIC but not used in our analysis. The seismic mass and radius directly translate to a surface gravity and a comparison between the seismic and KIC surface gravity is shown in the bottom panel of Fig. 13. Here we found a systematic difference indicated by a linear fit and binned values. For the red-clump stars (around $\log g=2.5$ ) the difference is negligible but drifts apart for stars above (towards lower surface gravity) and below (towards higher surface gravity) the red clump. The difference is, however, less than 0.5 dex for the entire range, and therefore within the uncertainties for the KIC parameters.

We also compared our seismic $\log g$ values for a few stars in common with the spectroscopic study of Bruntt et al. (2010, A\&A, in prep.). There is very good agreement with a mean difference (spectroscopy minus seismic $\log g$ ) of $\Delta \log g=+0.03 \pm$ 0.15 . We quote the rms scatter for the seven stars in common.

\section{Summary and conclusions}

We have analysed high-precision photometric time series from the first four months of Kepler observations and found more than 1000 stars that show a clear power excess in a frequency range typical for solar-type oscillations in red giants. We have applied robust and automated methods to accurately determine the global seismic parameters $v_{\max }$ and $\Delta v$, and provide an automated identification of the mode degree as well as small spacings 
for about one half of our sample. We have analysed the uncertainties in our parameter determination and find a clear relation for the uncertainty of $v_{\max }$ depending on the frequency resolution but also on the height-to-background ratio and the width of the power excess, which are largely determined by the star itself as long as the power excess is well above the white noise of the observations. We have applied our methods to solar data to determine solar reference values for $v_{\max }$ and $\Delta v$. The measured seismic parameters were then compared to those of an extensive multi-metallicity grid of stellar models, where the seismic parameters of the models were determined from scaling relations. A Bayesian approach for the search of a best fit between observed and model parameters allowed us to derive realistic uncertainties for all fundamental parameters. In principle, we could have estimated the fundamental parameters from the seismic scaling relations using the KIC temperatures. However, we found strong indications that our analysis produced more accurate fundamental parameters and gives us a more detailed view of the stellar populations in our sample of stars.

We have placed the analysed stars in a H-R diagram and found clear features in the distribution of the stars, which we identified as the red clump, the secondary clump, and the red bump. We found a mass gradient in the red clump with the lowmass stars accumulated at the bottom of the red clump and that the red bump is dominated by $1 M_{\odot}$ stars. Although we cannot determine the chemical composition reliably of individual stars we can conclude in a statistical sense that most of the red-clump stars in our sample are similar to the Sun in terms of the their initial chemical composition with some indications for a metallicity gradient that follows the mass gradient of the red clump. On the other hand we found that the bump stars are more consistent with metal-enhanced stars, which is surprising for a sample of stars that is selected according to criteria that do not constrain the chemical composition. A possible explanation could be that the mixing length parameter used to construct the models is too high. A slightly less efficient convection would shift the solar metallicity red-bump models towards lower temperatures in the direction of the observed red bump.

The large sample allowed us to investigate the detailed structure of the radius and mass distribution of red giants in the Kepler field of view clearly showing the different populations. A comparison with synthetic distributions indicated quantitative agreement but needs further investigations. With the present study and what was presented by Huber et al. (2010) and Mosser et al. (2010) there are now detailed red-giant populations available for three different regions in the sky, which should be used for future detailed population synthesis studies as first done by Miglio et al. (2009).

Finally, we mention that although the parameters uncertainties in our analysis are already quite realistic they still represent only a lower limit. There are several effects we do not yet take into account. For example, the frequency dependence of the large frequency spacing (cf. Mosser et al. 2010) might play a role, as might the weak asymmetry of the power excess humps. To investigate this in more detail we have to wait until Kepler can provide significantly longer time series. On the other hand, we expect a larger effect from the limitations of the used model grid. Although our grid covers a wide range in chemical composition, which is one of the parameters that can significantly influence the $M-L-R-T_{\text {eff }}$ relation, there are other model parameters such as overshooting or a better description of convection, that could change this relation as well and therefore could have consequences to our analysis. These effects are difficult to estimate and are still largely unexplored territory.
For the individual seismic parameters we refer to Hekker et al. (2010c) providing an online table for all red giants observed with Kepler. However, our fundamental parameter estimates are not included in this table because they will continuously be improved with the ongoing observations. But we encourage everybody who is interested in our results to request them personally from the lead author.

Acknowledgements. Funding for the Kepler Mission is provided by NASA's Science Mission Directorate. T.K. is supported by the Canadian Space Agency, the Austrian Research Promotion Agency, and the Austrian Science Fund. S.H., Y.P.E. and W.J.C. acknowledge support by the UK Science and Technology Facilities Council. D.H. acknowledges support by the Astronomical Society of Australia. The research leading to these results has received funding from the European Research Council under the European Community's Seventh Framework Programme (FP7/2007-2013)/ERC grant agreement no 227224 (PROSPERITY), as well as from the Research Council of K.U. Leuven grant agreement GOA/2008/04. The National Center for Atmospheric Research is a federally funded research and development center sponsored by the US National Science Foundation. We acknowledge support from the Australian Research Council. The authors gratefully acknowledge the Kepler Science Team and all those who have contributed to making the Kepler Mission possible.

\section{References}

Ballot, J., García, R. A., \& Lambert, P. 2006, MNRAS, 369, 1281

Barban, C., Matthews, J. M., De Ridder, J., et al. 2007, A\&A, 468, 1033

Basu, S., Chaplin, W. J., \& Elsworth, Y. 2010, ApJ, 710, 1596

Batalha, N. M., Borucki, W. J., Koch, D. G., et al. 2010, ApJ, 713, L109

Bedding, T. R., Huber, D., Stello, D., et al. 2010, ApJ, 713, L176

Borucki, W., Koch, D., Basri, G., et al. 2008, ed. Y.-S. Sun, S. Ferraz-Mello, \& J.-L. Zhou, IAU Symp., 249, 17

Borucki, W. J., Koch, D., Basri, G., et al. 2010, Science, 327, 977

Broomhall, A., Chaplin, W. J., Davies, G. R., et al. 2009, MNRAS, 396, L100

Brown, T. M., Gilliland, R. L., Noyes, R. W., \& Ramsey, L. W. 1991, ApJ, 368, 599

Buzasi, D., Catanzarite, J., Laher, R., et al. 2000, ApJL, 532, 133

Carrier, F., De Ridder, J., Baudin, F., et al. 2010, A\&A, 509, A73

Christensen-Dalsgaard, J. 2004, Solar Phys., 220, 137

Christensen-Dalsgaard, J., Kjeldsen, H., Brown, T. M., et al. 2010, ApJ, 713, L164

Creevey, O. L., Monteiro, M. J. P. F. G., Metcalfe, T. S., et al. 2007, ApJ, 659, 616

De Ridder, J., Barban, C., Carrier, F., et al. 2006, A\&A, 448, 689

De Ridder, J., Barban, C., Baudin, F., et al. 2009, Nature, 459, 398

Dupret, M., Belkacem, K., Samadi, R., et al. 2009, A\&A, 506, 57

Edmonds, P. D., \& Gilliland, R. L. 1996, ApJ, 464, L157

Frandsen, S., Carrier, F., Aerts, C., et al. 2002, A\&A, 394, L5

Frohlich, C., Andersen, B. N., Appourchaux, T., et al. 1997, Solar Phys., 170, 1

Gilliland, R. L., Brown, T. M., Christensen-Dalsgaard, J., et al. 2010, PASP, 122, 131

Girardi, L. 1999, MNRAS, 308, 818

Girardi, L., Groenewegen, M. A. T., Hatziminaoglou, E., \& da Costa, L. 2005, A\&A, 436, 895

Gizon, L., \& Solanki, S. K. 2003, ApJ, 589, 1009

Gruberbauer, M., Kallinger, T., Weiss, W. W., \& Guenther, D. B. 2009, A\&A, 506, 1043

Harvey, J. 1985, in Future Missions in Solar, Heliospheric \& Space Plasma Physics, ed. E. Rolfe \& B. Battrick, ESA SP, 235, 199

Hekker, S., Kallinger, T., Baudin, F., et al. 2009, A\&A, 506, 465

Hekker, S., Broomhall, A., Chaplin, W. J., et al. 2010a, MNRAS, 402, 2049

Hekker, S., Debosscher, J., Huber, D., et al. 2010b, ApJ, 713, L187

Hekker, S., Elsworth, Y., De Ridder, J., et al. 2010c, A\&A, accepted

Huber, D., Bedding, T. R., Stello, D., et al. 2010, ApJ, accepted

[arXiv: 1010.4566]

Huber, D., Stello, D., Bedding, T. R., et al. 2009, Commun. Asteroseismol., 160, 74

Jenkins, J. M., Caldwell, D. A., Chandrasekaran, H., et al. 2010, ApJ, 713, L120 Kallinger, T., Guenther, D. B., Matthews, J. M., et al. 2008a, A\&A, 478, 497

Kallinger, T., Guenther, D. B., Weiss, W. W., et al. 2008b, CoAst, 153, 84 Kallinger, T., Weiss, W. W., Barban, C., et al. 2010, A\&A, 509, A77

Kallinger, T., Zwintz, K., Pamyatnykh, A. A., Guenther, D. B., \& Weiss, W. W. 2005, A\&A, 433, 267

Kjeldsen, H., \& Bedding, T. R. 1995, A\&A, 293, 87

Kjeldsen, H., Bedding, T. R., \& Christensen-Dalsgaard, J. 2008, ApJ, 683, L175 Latham, D. W., Brown, T. M., Monet, D. G., et al. 2005, in BAAS, 37, 1340 
Mathur, S., García, R. A., Régulo, C., et al. 2010, A\&A, 511, A46

Mazumdar, A., Mérand, A., Demarque, P., et al. 2009, A\&A, 503, 521

Merline, W. J. 1999, in Precise Stellar Radial Velocities, ed. J. B. Hearnshaw, \&

C. D. Scarfe, ASP Conf. Ser., 185, IAU Colloq., 170, 187

Miglio, A., Montalbán, J., Baudin, F., et al. 2009, A\&A, 503, L21

Monet, D. G., Jenkins, J. M., Dunham, E. W., et al. 2010, ApJL, submitted [arXiv: 1001.0305 ]

Mosser, B., \& Appourchaux, T. 2009, A\&A, 508, 877

Mosser, B., Belkacem, K., Goupil, M. J., et al. 2010, A\&A, 517, A22

Nigam, R., Kosovichev, A. G., Scherrer, P. H., \& Schou, J. 1998, ApJL, 495, 115

Pietrinferni, A., Cassisi, S., Salaris, M., \& Castelli, F. 2004, ApJ, 612, 168

Retter, A., Bedding, T. R., Buzasi, D. L., Kjeldsen, H., \& Kiss, L. L. 2003, ApJ, 591, L151
Stello, D., \& Gilliland, R. L. 2009, ApJ, 700, 949

Stello, D., Bruntt, H., Kjeldsen, H., et al. 2007, MNRAS, 377, 584

Stello, D., Bruntt, H., Preston, H., \& Buzasi, D. 2008, ApJ, 674, L53

Stello, D., Chaplin, W. J., Basu, S., Elsworth, Y., \& Bedding, T. R. 2009a, MNRAS, 400, L80

Stello, D., Chaplin, W. J., Bruntt, H., et al. 2009b, ApJ, 700, 1589

Stello, D., Basu, S., Bruntt, H., et al. 2010, ApJ, 713, L182

Tarrant, N. J., Chaplin, W. J., Elsworth, Y., Spreckley, S. A., \& Stevens, I. R. 2007, MNRAS, 382, L48

Tassoul, M. 1980, ApJS, 43, 469

Toutain, T., \& Froehlich, C. 1992, A\&A, 257, 287

van Leeuwen, F. 2007, A\&A, 474, 653 